\title{
Bandwidth Enhancement Technique for Bipolar Single Stage Distributed Amplifier Design
}

\author{
Temitope Odedeyi ${ }^{1}$, Izzat Darwazeh ${ }^{2}$ \\ Department of Electronic and Electrical Engineering, University College London \\ London, United Kingdom \\ ${ }^{1}$ temitope.odedeyi.13@ucl.ac.uk, \\ 2i.darwazeh@ucl.ac.uk
}

\begin{abstract}
This work reports a novel approach to extending the bandwidth of single stage distributed amplifiers (SSDAs). The three-stepped technique involves scaling down the inductance on the input artificial transmission line (ATL); creating a high frequency resonance peak by the addition of shunt capacitance on the input ATL; and compensating for the resulting increased reflection with adapted negative resistance attenuation compensation techniques. Compared with the inductive-peaked cascode technique applied in the SSDA which currently has the highest reported bandwidth, simulation results, based on full foundry transistor models, predict up to $30 \%$ improvement in gainbandwidth (GBW) performance for the same active device at the same bias. In addition, the reduction in the length of the input ATL effectively reduces transmission line losses, thereby improving the overall gain performance.
\end{abstract}

Index Terms-Single stage distributed amplifier (SSDA); transmission line scaling, attenuation compensation; Indium Phosphide (InP); double heterojunction bipolar transistor (DHBT); inductive peaking

\section{INTRODUCTION}

Distributed amplification was initially proposed as a means of improving the robustness of thermionic valve circuits in terms of bandwidth and bias voltage [1]. This improvement is achieved by separating the inter-electrode capacitance of individual vacuum tubes while adding their transconductance. Since its introduction, the concept has been applied in the design of amplifiers with very broad bandwidth; the highest reported being $235 \mathrm{GHz}$ using a transistor with $350 \mathrm{GHz}$ transition frequency $\left(f_{T}\right)$ [2].

To achieve substantial gain, the distributed amplifier (DA) was conceived as a multistage amplifier [1], [3]. The concept and practicability of single stage distributed amplifiers (SSDAs) was demonstrated in [4]. Furthermore, it has been established that due to the multiplicative gain achieved from cascading two or more SSDAs with moderate gain; higher gain can be achieved than with a conventional DA having the same number of gain cells [5]. This has made the cascaded SSDA (CSSDA) a preferred option for high gain wideband amplifiers.

Bipolar transistors are attractive for DA designs because they offer higher gains and better linearity than field effect transistors (FETs) of a similar generation[6]. However, the

The authors would like to acknowledge the Petroleum Technology Development Fund (PTDF) of Nigeria for sponsoring Temitope Odedeyi's PhD program. resistive input characteristics of bipolar transistors directly translates to higher losses on their input artificial transmission line (ATL) and to a complex characteristic impedance that makes the design of line termination more challenging [7]. This effectively introduces higher attenuation with each additional stage of the DA, meaning that fewer stages can be realised before the overall gain begins to drop [7], [8]. These considerations make bipolar transistors better suited in SSDAs than in multistage distributed amplifiers.

Several techniques have been proposed to extend the bandwidth of distributed amplifiers, including those which employ bipolar transistors [9], [10], [11], [12]. The work presented here extends such techniques to achieve further bandwith improvement using ATL optimization methods.

The outline of this paper is as follows: section II describes the fundamental design concept as a three step process: increasing the $3 \mathrm{~dB}$ cutoff frequency of the input line by scaling down the line length; introducing a high frequency peak through a shunt capacitance on the input line; and applying attenuation compensation on the output line to improve overall gain profile. Section III demonstrates the applicability of this concept through simulation studies based on full circuit models of an Indium Phosphide InP double heterojuntion bipolar transistor (DHBT) and compares the bandwidth and gain performance of the new technique to the technique adopted in the SSDA with the widest reported bandwidth [2]. Section IV concludes the paper.

\section{DESIGN CONCEPT}

Fig. 1 shows simplified equivalent circuits of the input and output ATLs of an HBT SSDA. The lines are made up of intrinsic capacitance $C_{\pi}$ and $C_{c e}$ and inductive lines $L_{B}$ and $L_{C}$; with both lines coupled by the transistor transconductance $g_{m} . r_{\pi}$ and $r_{o}$ are the transistor base-emitter junction resistance and output, resistance respectively and $R_{\mathrm{TERM}}$ is the terminating resistance.

The design technique proposed and verified in this paper is a three-stepped process involving two modifications to the input line of the conventional SSDA; and one to the output line.

\section{A. Input Line Modification}

It has been established that the bandwidth performance of a DA is determined by the cutoff frequency of the input line [13], 


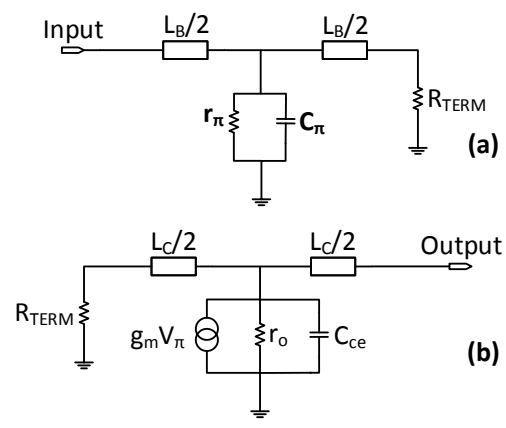

Fig. 1. Simplified equivalent circuits of (a) input transmission line of a conventional HBT SSDA and (b) Output Transmission Line of a conventional HBT SSDA.

[3]. This is due to the fact that the input capacitances of both bipolar and field effect transistors is usually larger than their output capacitances. Hence, by the image impedance design approach employed in conventional DA, the input line would have a lower cutoff frequency which will dominate the DA bandwidth performance.

Therefore, to achieve higher bandwidth, the cutoff frequency of the input artificial transmission line (ATL) must be increased. This is achieved in two steps:

Step 1: Increase the $3 d B$ cutoff frequency of input line by scaling down the line length (inductor value, if discrete components are used): Recall that the image impedance of the input transmission line $\left(Z_{o-i n}\right)$ is given by

$$
Z_{o-i n}=\sqrt{\frac{L_{B}}{C_{\pi}}\left(1-\frac{4 \pi^{2} f^{2} L_{B} C_{\pi}}{4}\right)}
$$

with $C_{\pi}$ being the base-emitter capacitance of the common emitter amplifier; $L_{B}$ being the inductance value required to achieve the distributed effect; and $\omega$ being the angular frequency. With the cutoff frequency of the line, $f_{c}=$ $1 / \pi \sqrt{L_{B} C_{\pi}}$ (for ideal lossless lines). At this frequency, the image impedance transitions from being purely real to being purely imaginary if the line is terminated with the DC value of $Z_{o-i n}$, denoted by $Z_{o-i n}^{*}$ such that

$$
Z_{o-i n}^{*}=\sqrt{\frac{L_{B}}{C_{\pi}}} .
$$

We extend the cutoff frequency by scaling $L_{B}$ by a factor, $\zeta$ (with $\zeta<1$ ), while keeping the terminating impedances $R_{T E R M}$ at the value of $Z_{o-i n}^{*}$. This results in cut-off frequency $f_{c^{\prime}}$ that is

$$
\frac{1}{\pi \sqrt{L_{B} C_{\pi}}}<f_{c^{\prime}}<\frac{1}{\pi \sqrt{\zeta L_{B} C_{\pi}}} .
$$

For $f_{c^{\prime}}=1 / \pi \sqrt{\zeta L_{B} C_{\pi}}$, the terminating impedance must be reduced such that $R_{T E R M}=\sqrt{\zeta L_{B} / C_{\pi}}$. However, this would lead to a corresponding reduction in gain, as

$$
\text { Gain }=N g_{m} \frac{\sqrt{Z_{o-i n} \cdot Z_{o-o u t}}}{2},
$$

where $g_{m}$ is the transistor transconductance; $Z_{o-i n}$ is the input line impedance; $Z_{o-o u t}$ is the output line impedance and $N$ is

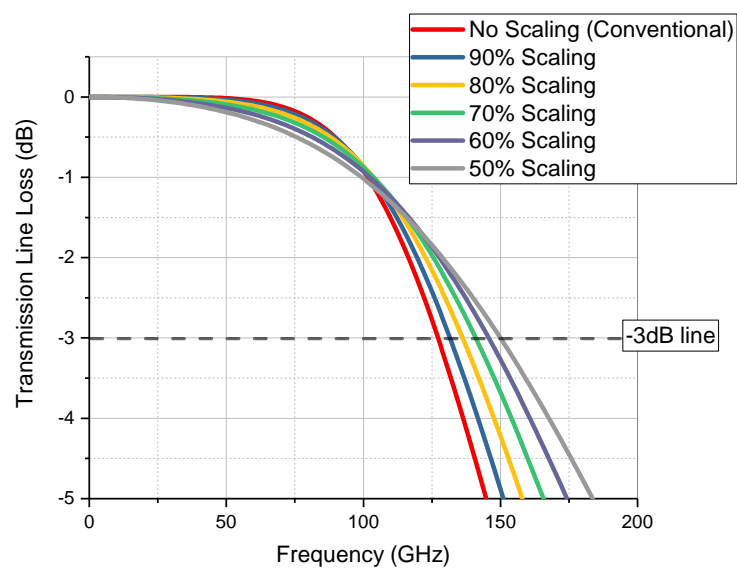

Fig. 2. Bandwidth improvement from line scaling.

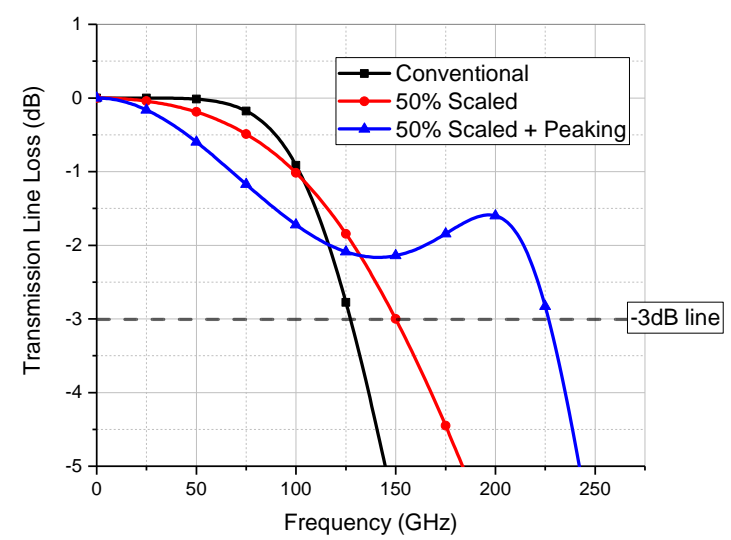

Fig. 3. Comparison of line losses for a conventional transmission line, 50\% scaled transmission line and a $50 \%$ scaled transmission line with peaking.

the number of gain stages. By terminating with $Z_{o-i n}^{*}$, the gain is kept at the same level as will be obtained from a conventional DA.

Fig. 2 shows the gain in bandwidth with incremental scaling for a hypothetical HBT with $C_{\pi}$ of $50 \mathrm{fF}$ and $50 \Omega$ termination.

Step 2: Introduce a high frequency peak through a shunt capacitance: The addition of shunt capacitance $C_{\text {peak }}$ at the input line would result in an LC resonant peak given to a good approximation by

$$
f_{\text {peak }} \approx \frac{1}{\pi \sqrt{\zeta \frac{L_{B}}{2}\left(C_{\pi}+C_{\text {peak }}\right)}} .
$$

By optimising the value of $C_{\text {peak }}$ and $\zeta$, the peak frequency, amplitude and bandwidth can be used to significantly improve the $3 \mathrm{~dB}$ bandwidth of the input line.

To illustrate the concept, Fig. 3 compares the bandwidth of a conventional image impedance line with a $50 \%$ scaled line and a line which features both $50 \%$ scaling and LC peaking from an hypothetical HBT with $C_{\pi}$ of $50 \mathrm{fF}$ and $50 \Omega$ termination. This is shown in terms of transmission loss of three different ATLs. 


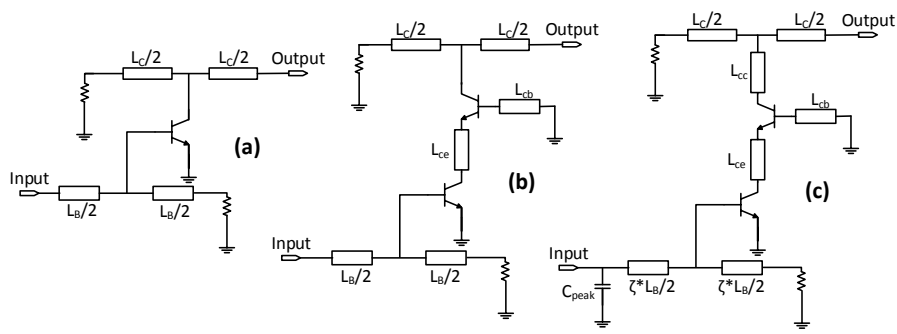

Fig. 4. Schematic circuits of (a) conventional SSDA; (b) SSDA with inductive-peaked cascode [15]; and (c) new SSDA with scaled input line, shunt capacitance and adapted loss compensation.

\section{B. Output Line Modification}

Step 3: Apply negative resistance attenuation compensation technique to improve the overall gain profile: The effect of increasing the ratio of $C_{\pi}$ to $L_{B}$ is that the transmission line would have higher reflection at high frequencies leading to a dip in the gain profile. To counteract this, the HBT version of the attenuation compensation techniques described in [11] and [10] is modified to achieve a flat gain profile.

The key feature of this technique is the introduction of two additional transmission lines, $L_{c b}$ (at the base of the common base transistor) and $L_{c e}$ (between the common emitter and common base transistors) to a cascode gain cell. For this circuit, the output impedance $Z_{\text {out }}$ is

$$
\begin{aligned}
Z_{\text {out }}=\frac{\left(Z_{c e}+j \omega L_{c e}\right)}{\left(Z_{c e}+\right.} & \left.j \omega L_{c e}\right)+\left(Z_{b e}+j \omega L_{c b}\right) \\
& \times\left(\frac{g_{m} Z_{c e}}{j \omega C_{b e}}+\left(Z_{b e}+j \omega L_{c b}\right)\right)+Z_{c e}
\end{aligned}
$$

where $\mathrm{Z}_{c e}$ is the impedance between the collector and the emitter of the common collector and $Z_{b e}$ is the impedance between the base and the emitter of the common base forming the cascode pair. The value of $L_{c e}$ is adjusted proportional to $\zeta$ to increase the negative resistance generated in the circuit and maintain the desired high frequency gain. The resulting inductance value, is denoted by $L_{c e}^{\prime}$ in (7). $L_{c b}$ is also modified appropriately to maintain stability (the resulting value is denoted by $L_{c b}^{\prime}$ in (7)).

The resulting output impedance is given by

$$
\begin{aligned}
& Z_{\text {out }}=\frac{\left(Z_{c e}+j \omega L_{c e}^{\prime}\right)}{\left(Z_{c e}+j \omega L_{c e}^{\prime}\right)+\left(Z_{b e}+j \omega L_{c b}^{\prime}\right)} \\
& \times\left(\frac{g_{m} Z_{c e}}{j \omega C_{b e}}+\left(Z_{b e}+j \omega L_{c b}^{\prime}\right)\right)+Z_{c e}
\end{aligned}
$$

Fig. 4(a) shows the schematic circuit for a conventional SSDA; Fig. 4(b) shows the loss compensation circuit presented in [14]; while Fig. 4(c) shows the new SSDA schematic featuring the scaled input line, the shunt capacitance for input line peaking and the adapted loss compensation for gain flatness.

Due to the complexity of the analysis involved, the optimal values for $L_{c b}^{\prime}$ and $L_{c e}^{\prime}$ may be more easily obtained through computer aided optimisation. For extra degree of tunability, an

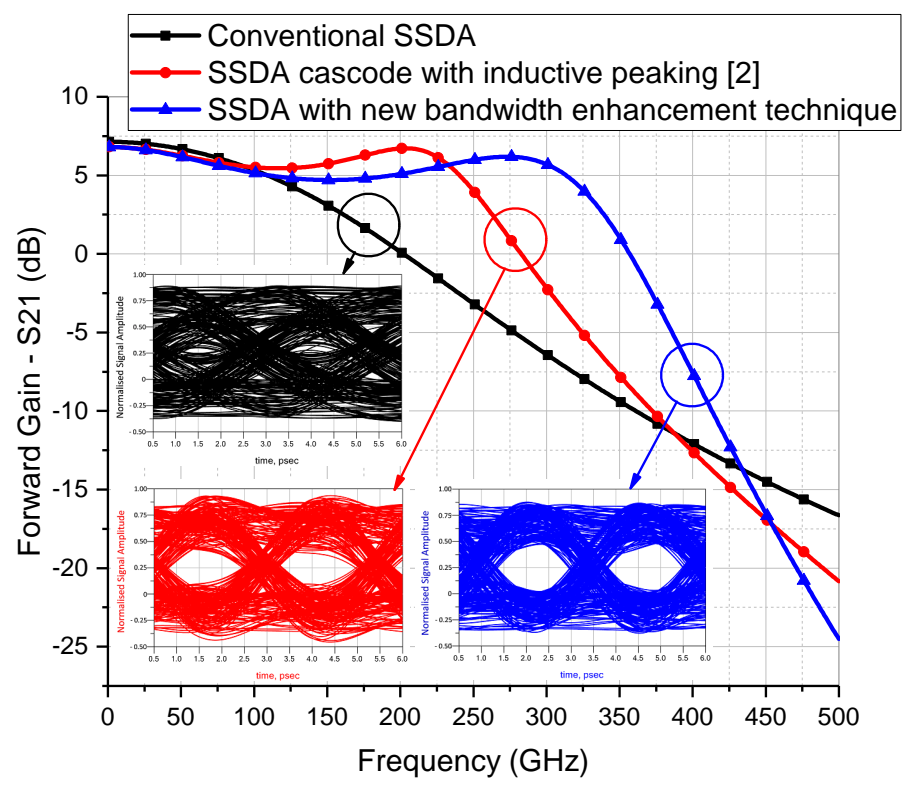

Fig. 5. Forward Gain $\mathrm{S} 21(\mathrm{~dB})$ with corresponding simulated eye diagrams at 500Gbit/s (inset): Conventional - 3dB-bandwidth $132 \mathrm{GHz} / 7 \mathrm{~dB}$ gain; SSDA with inductive-peaked cascode - $3 \mathrm{~dB}$-bandwidth $253 \mathrm{GHz} / 7 \mathrm{~dB}$ gain; and SSDA with new bandwidth enhancement technique - 3dB-bandwidth $328 \mathrm{GHz} / 7 \mathrm{~dB}$ gain.

inductance $L_{c c}$ is added between the collector terminal of the common base transistor and line $L_{C}$.

It is interesting to note that either of steps 1 or 2 may be combined with output line attenuation compensation to achieve improved bandwidth performance. However combining the two steps expectedly yields the optimum result of maximum bandwidth.

\section{PerformanCE ASSESSMENT AND VERIFICATION}

The aforementioned technique is demonstrated in simulation using the full circuit model of TSC250; an Indium Phosphide foundry heterojunction bipolar transistor process by Teledyne Scientific Companies. The device offers $f_{t} / f_{\max }$ of $350 / 600$ $\mathrm{GHz}$ while maintaining a common-emitter breakdown voltage of more than $4 \mathrm{~V}$. Detailed descriptions of this process is available in [16].

Figures 5, 6 and 7 compare the forward gain (S21), the input line reflection (S11) and the output line reflection (S22) respectively, of a conventional SSDA (Fig. 4a); SSDA with the inductive-peaked cascode technique used in [15] (Fig. 4b); and a SSDA that features the proposed bandwidth extension technique (Fig. 4c). For fair comparison, all three circuits are simulated using the same process model and operate at the same bias conditions. Lossless transmission lines and ideal circuit elements were used in simulations.

Circuit simulations predict a $328 \mathrm{GHz} 3 \mathrm{~dB}$ bandwidth for the the new technique. This is nearly two-and-a-half times the bandwidth of an otherwise equivalent SSDA designed using conventional means and 30\% higher than what is achieved with the technique adopted in [15] (Fig. 5). The input line modifications result in a gain ripple of $\pm 1 \mathrm{~dB}$ (Fig. 5). The phase response of the new design was tested and shows linear 


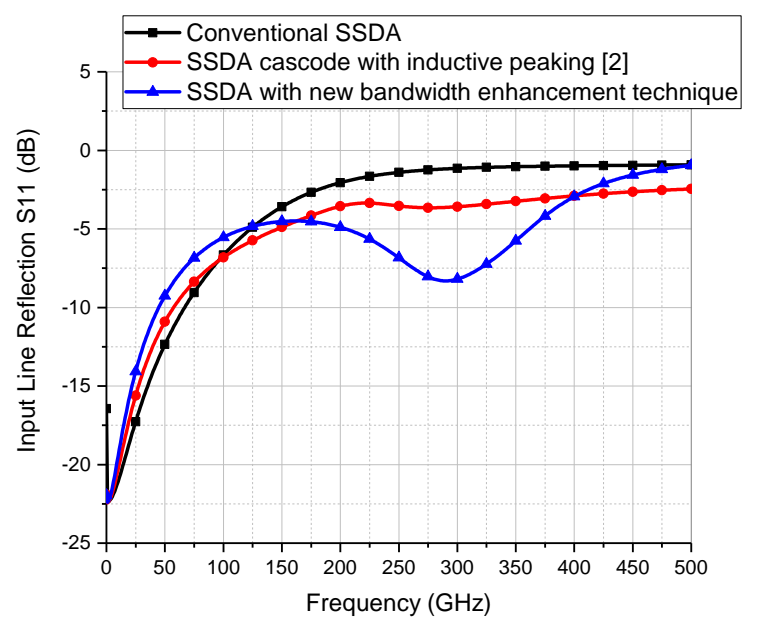

Fig. 6. Input Line Reflection S11(dB) Comparison.

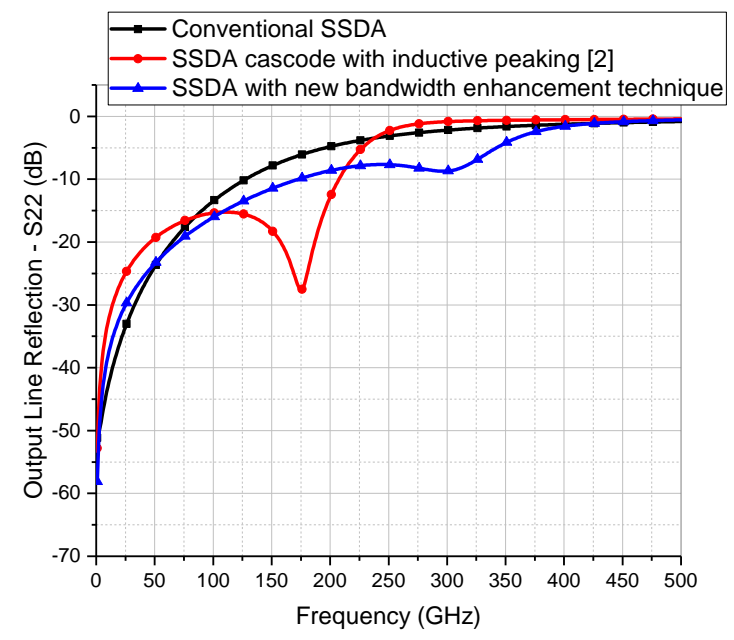

Fig. 7. Output Line Reflection S22(dB) Comparison.

phase up to the $3 \mathrm{~dB}$ gain of the amplifier, leading to good pulse response which is indicated by an open eye shown in the inset of the figure and simulated assuming $500 \mathrm{Gbit} / \mathrm{s}$ data stream of $2^{12}-1$ pseudo random binary sequence. The open eye for the new design furthermore show its advantage when compared to the fully closed eye diagram of the conventional design and the less open one of the design following [15].

Additionally, the design improves the input and output matching behaviour as shown, respectively in Figs. 6 and 7. The design is unconditionally stable, as both the EdwardsSinsky stability parameter $(\mu)$ and the Rollett's stability factor $(K)$ are greater than 1 from DC up to $500 \mathrm{GHz}$. Furthermore, the new design displays better phase linearity and smaller delay variation than the conventional design. The group delay is simulated to be approximately $4 \pm 1$ ps from low frequencies to $500 \mathrm{GHz}$, which leads to the good pulse response observed in the eye diagram of Fig. 5. The power consumption of the amplifier is expected to be below $12 \mathrm{~mW}$.

The transmission line scaling technique has the added advantage of reducing the attenuation associated with transmission lines, however, it is noteworthy that this technique will not be well suited to multi-stage distributed amplifiers as the effects of improper distribution from line scaling become more pronounced with additional stages. To meet higher gain requirement, SSDAs based on this bandwidth enhancement technique can be suitably applied as units of high-gain-block wideband amplifiers through cascading or in a single stage matrix topology.

\section{CONCLUSION}

A new design technique for bipolar based SSDAs is introduced through which significant improvement in bandwidth can be achieved. It involves purposely scaling down the inductance and introducing a high frequency LC peak (through a shunt capacitance) on the amplifier's input line; and negative resistance attenuation compensation on the output line. This technique can be used to achieve distributed amplifiers with significantly wider bandwidth and with improved time domain response and input and output matching behaviour.

\section{REFERENCES}

[1] WS Percival. Thermionic valve circuits. British patent, 460562:25, 1937.

[2] Klas Eriksson, Izzat Darwazeh, and Herbert Zirath. InP DHBT distributed amplifiers with up to $235-\mathrm{GHz}$ bandwidth. IEEE Transactions on Microwave Theory and Techniques, 63(4):1334-1341, 2015.

[3] Edward L Ginzton, William R Hewlett, John H Jasberg, and Jerre D Noe. Distributed amplification. Proceedings of the IRE, 36(8):956-969, 1948.

[4] G Nwaogu and CS Aitchison. A very broadband microwave distributed amplifier using FETs. In 1981 11th European Microwave Conference, pages 609-613, 1981.

[5] J. Y. Liang and C. S. Aitchison. Gain performance of cascade of single stage distributed amplifiers [microwave circuits]. Electronics Letters, 31(15):1260-1261, Jul 1995

[6] ME Kim, AK Oki, GM Gorman, DK Umemoto, and JB Camou. GaAs heterojunction bipolar transistor device and IC technology for highperformance analog and microwave applications. IEEE Transactions on Microwave Theory and Techniques, 37(9):1286-1303, 1989.

[7] A Iqbal and IZ Darwazeh. Analytical modelling of the heterojunction bipolar transistor based distributed amplifier. In High Frequency Postgraduate Student Colloquium, 1996., 2nd, pages 11-16. IEEE, 1996.

[8] A Iqbal and IZ Darwazeh. A $23 \mathrm{GHz}$ baseband HBT distributed amplifier for optical communication systems. In Microwave Conference, 1998. 28th European, volume 1, pages 6-11. IEEE, 1998.

[9] Claudio Paoloni and S Kosslowski. New approach on the design of travelling wave amplifier. In Circuits and Systems, 1991., Proceedings of the 34th Midwest Symposium on, pages 414-417. IEEE, 1991.

[10] Kevin W Kobayashi, Reza Esfandiari, and Aaron K Oki. A novel HBT distributed amplifier design topology based on attenuation compensation techniques. IEEE transactions on microwave theory and techniques, 42(12):2583-2589, 1994.

[11] Shunji Kimura, Yuhki Imai, Yohtaro Umeda, and Takatomo Enoki. Losscompensated distributed baseband amplifier ic's for optical transmission systems. IEEE transactions on microwave theory and techniques, 44(10):1688-1693, 1996.

[12] J-P Rooney, Richard Parry, Ian Hunter, and Roger D Pollard. A filter synthesis technique applied to the design of multistage broad-band microwave amplifiers. IEEE transactions on microwave theory and techniques, 50(12):2947-2953, 2002.

[13] Thomas TY Wong. Fundamentals of distributed amplification. Artech House, 1993.

[14] Shunji Kimura and Yuhki Imai. 0-40 GHz GaAs MESFET distributed baseband amplifier ICs for high-speed optical transmission. IEEE transactions on microwave theory and techniques, 44(11):2076-82, 1996.

[15] Klas Eriksson, Izzat Darwazeh, and Herbert Zirath. InP DHBT wideband amplifiers with up to $235 \mathrm{GHz}$ bandwidth. In 2014 IEEE MTT-S International Microwave Symposium (IMS2014), pages 1-4. IEEE, 2014.

[16] Jonathan Hacker, Munkyo Seo, Adam Young, Zach Griffith, Miguel Urteaga, Thomas Reed, and Mark Rodwell. THz MMICs based on InP HBT technology. In Microwave Symposium Digest (MTT), 2010 IEEE MTT-S International, pages 1126-1129. IEEE, 2010. 